\title{
REMARKS ON THE STABILITY OF REVERSE HÖLDER INEQUALITIES AND QUASICONFORMAL MAPPINGS
}

\author{
B. BOJARSKI
}

In this note we indicate that a refined version of the local Fefferman-Stein inequality for a sharp maximal operator improves the information on the local integrability of functions satisfying the reverse Hölder inequality. In particular, this implies better estimates than previously achieved by similar methods ([7], [8]) for the order of integrability of derivatives of a $K$-quasiconformal mapping as the dilatation $K \rightarrow 1$. These considerations suggest some intriguing and urgent problems on the border of real analysis and quasiconformal theory.

Let $\Omega$ be a domain in $\boldsymbol{R}^{n}$. A non-negative measurable function $f(x), x \in \Omega$, is said to satisfy the reverse Hölder inequality if for some constants $p, q, p>q$, the inequality

$$
\left(f_{Q} f^{p} d x\right)^{1 / p} \leqq K\left(f_{Q} f^{q} d x\right)^{1 / q}
$$

holds for all cubes $Q \subset \subset \Omega$ and some constant $K$ (depending on $f$ ). Hereafter we consider only cubes with edges parallel to coordinate axes of $\boldsymbol{R}^{n}$. The barred integral $f_{Q} f d x$ denotes the average

$$
f_{Q} f d x=\frac{1}{|Q|} \int_{Q} f d x \equiv f_{Q}
$$

If (1) holds with $p, q$ and $K$ fixed, then we write $f \in B_{q}^{p}(K)$. For $p$ positive, the condition (1) specifies a subclass $B_{q}^{p}(K)$ of $L_{\mathrm{loc}}^{p}(\Omega) . B_{q}^{p}(K)$ is not a (linear) subspace of $L_{\mathrm{loc}}^{p}(\Omega)$. The inequality (1) expresses some balance between small and big values of $f$ measured by the averages $\left(f_{Q} f^{p} d x\right)^{1 / p}$. It excludes "big" local oscillations of $f$. Thus it is a form of the averaged Harnack property. In the extreme case $p=+\infty, q=-\infty$ (1) reduces to the uniform local Harnack inequality:

$$
\max _{Q} f \leqq K \inf _{Q} f \text { for each } Q \subset \subset \Omega .
$$

In the non-trivial case $K>1$, since otherwise, by the Hölder inequality, $f \equiv$ const a.e.

If $f$ is a weight, i.e., a positive, locally integrable measurable function, then the union $\bigcup_{K \geqq 1} B_{1 /(1-p)}^{1}(K), p>1$, is the famous Muckenhoupt class $A_{p}$, [9]. F. Gehring in [4] considers the class $B_{1}^{\delta}=\bigcup_{K \geqq 1} B_{1}^{\delta}(K)$ for some $\delta>1$. Muckenhoupt and 
Gehring show that if $f \in A_{p}$, then actually $f \in A_{p-\varepsilon}$ for some $\varepsilon>0$, depending on $f$ in general. Let us recall the precise formulation of Gehring's important lemma:

Lemma 1. Let $\Omega$ be a domain in $\boldsymbol{R}^{n}, q>1, f \in L_{\mathrm{loc}}^{q}(\Omega)$. Suppose that for each cube $Q \subset \Omega$ the inequality

$$
\left(f_{Q}|f|^{q} d x\right)^{1 / q} \leqq K f_{Q}|f| d x
$$

holds with a constant $K \geqq 1$ independent of $Q$. Then there exist a $p=p(n, q, K)>q$ and $C=C(n, q, K) \geqq 1$ such that

$$
\left(f_{Q}|f|^{p} d x\right)^{1 / p} \leqq C\left(f_{Q}|f|^{q} d x\right)^{1 / q} .
$$

In particular, $f \in L_{\mathrm{loc}}^{p}(\Omega)$ for some $p>q$.

More generally, $f \in B_{q}^{p} \Rightarrow f \in B_{q-\varepsilon}^{p+\varepsilon}, \varepsilon>0$, if $B_{q}^{p}=\cup_{K} B_{q}^{p}(K)$. This is the "selfimproving" property of reverse Hölder inequalities. Very little is known about the possible (optimal) choice of $p(n, q, K)$.

The stability problem for reverse Hölder inequalities is the problem of studying how the (maximal) exponent of summability of $f$ in $B_{q}^{p}(K)$ increases with $K \rightarrow 1$. In the extremal case $K=1, f$ is constant, thus in $L_{\text {loc }}^{\infty}(\Omega)$.

It was recognized in [1] that the stability problem for reverse Hölder inequalities is intimately related to the study of subclasses of functions in $L_{\mathrm{loc}}^{q}(\Omega), q \geqq 1$, satisfying the condition

$$
\left(f_{Q}\left|f-f_{Q}\right|^{q} d x\right)^{1 / q} \leqq \varepsilon f_{Q} \quad\left(f_{Q}=f_{Q}|f| d x\right)
$$

for each cube $Q \subset \Omega$. The inequality (2) for the local averaged oscillations $f_{Q, p}^{\#}=$ $\left(\int_{Q}\left|f-f_{Q}\right|^{p} d x\right)^{1 / p}$ expresses the fact that the functions in these subclasses can be locally approximated by constants in some uniform way.

We are naturally led to consider the Fefferman-Stein sharp maximal operator (for $q=1$ )

$$
f^{\#}(x)=\sup _{Q} f_{Q}\left|f-f_{Q}\right| d x
$$

with the supremum taken over all cubes $Q$ contained in $\Omega$ and containing $x$. The inequality (2) has its "pointwise" version $(q=1)$

$$
f^{\#}(x) \leqq \varepsilon M f(x),
$$

where $M f$ is the Hardy-Littlewood nonsymmetric maximal function of $f$. (2) and $\left(2^{\prime}\right)$ are always true if $\varepsilon=2$ and $q=1$. The point is that, for $\varepsilon$ small, (2) and (2') contain very deep information expressed in Theorem 1 and Lemma 2.

The situation here exhibits some analogies to the stability properties of $K$-quasiconformal mappings as the dilatation $K \rightarrow 1$. By Liouville's theorem, 1-quasiconformal mappings reduce to Möbius transformations (in the space $\boldsymbol{R}^{n}$, 
$n \geqq 3$ ). By the Lavrent'ev-Reshetnyak theorem [10], [11], as $K \rightarrow 1$, a $K$-quasiconformal mapping, say of a ball $D$ in $\boldsymbol{R}^{n}$, can be uniformly approximated in the closed ball $D$ by a Möbius transformation. Moreover, the derivatives of the mapping are locally integrable with the exponent $p \rightarrow \infty$ as $K \rightarrow 1(p \sim c(n) /(K-1))$ and approximate the derivatives of the Möbius transformation in some integral sense.

Stability for reverse Hölder inequalities was first discussed in [1] (see also [7]). We sketch here a proof which uses the important

Theor em 1 (Reshetnyak and Gurov [5], [6], [11]). Let the (vector-valued) function $f: \Omega \rightarrow \boldsymbol{R}^{m}$ satisfy the inequality

$$
\left(f_{Q}\left|\hat{f}-f_{Q}\right|^{q} d x\right)^{1 / q} \leqq \varepsilon|f|_{Q}
$$

for each cube $Q \subset \Omega$ and a fixed $q \geqq 1$ (with the usual understanding of norm $f$ in $\left.\boldsymbol{R}^{m}\right)$. Then for some constant $C_{0}$, depending on $q$ and $n$ only, $f \in L_{\mathrm{loc}}^{p}(\Omega)$ for every $p<C_{0} / \varepsilon, p \geqq 1$. Moreover, for $p_{1}<p<C_{0} / \varepsilon$ the estimate

$$
\left(f_{Q}\left|f-f_{Q}\right|^{p} d x\right)^{1 / p} \leqq C_{2}\left(|f|_{Q}\right)^{1-q / p}\left(\varepsilon \log \frac{a}{\varepsilon}\right)^{1-q / p}\left(f_{Q}\left|f-f_{Q}\right|^{q} d x\right)^{1 / p}
$$

holds. The constants $C_{2}$ and $a$ depend on $q$ and $n$ only provided $\varepsilon$ is sufficiently small, $0<\varepsilon<\varepsilon_{0}(n, q)$.

To recall the idea from [1] we consider, for simplicity, only the case of $B_{1}^{2}(K)$, i.e.,

Then we have

$$
\left(f_{Q}|f|^{2} d x\right)^{1 / 2} \leqq K f_{Q}|f| d x .
$$

hence

$$
f_{Q}\left(|f|-|f|_{Q}\right)^{2} d x=f_{Q}|f|^{2} d x-|f|_{Q}^{2} \leqq\left(K^{2}-1\right)|f|_{Q}^{2} ;
$$

This implies

$$
\left.\left.f_{Q}|| f|-| f\right|_{Q}\left|d x \leqq\left(f_{Q}\left(|f|-|f|_{Q}\right)^{2} d x\right)^{1 / 2} \leqq \sqrt{K^{2}-1}\right| f\right|_{Q} .
$$

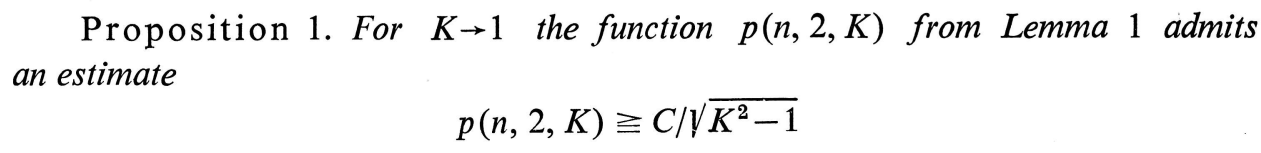

for some positive $C$ depending on $n$ only.

An analogous statement holds for the classes $B_{q}^{p}(K)$ for $K \rightarrow 1$. The class $B_{1}^{2}(K)$ can be considered a model case for the classes $B_{q}^{p}(K)$ since the inequality (1), by a series of elementary substitutions, is essentially reducible to (3).

Proposition 1 is complementary to Lemma 1 for $K \rightarrow 1$. Compared with Proposition 3 in [7], it gives stronger estimates for the integrability exponent $p$. The point is that in [7] Proposition 3 was presented as a corollary of the important Lemma 2 
in [7] valid in a much more general situation than described in the theorem of Gurov and Reshetnyak.

Lemma 2. Let $f \in L_{\mathrm{loc}}^{1}(\Omega), 0<\sigma \leqq 1,1 \leqq p<\infty$. Suppose that for each cube $Q \subset \Omega$ the inequality

$$
f_{\sigma Q}\left|f-f_{\sigma Q}\right| d y \leqq c_{1} f_{Q}|f| d y, \quad c_{1}=10^{-6 n p}
$$

holds. Then $f \in L_{\mathrm{loc}}^{p}(\Omega)$ and for each cube $Q \subset \subset \Omega$ we have

$$
\left(f_{1 / 2 Q}|f|^{p} d y\right)^{1 / p} \leqq c_{2} f_{Q}|f| d y, \quad c_{2}=\frac{10^{8 n^{2} p}}{\sigma^{n}},
$$

where $\sigma Q$ is the cube with the same center as $Q$, expanded by the factor $\sigma$.

The precise numerical values of the constants $c_{1}$ and $c_{2}$ are not essential, since these values are far from optimal. The choice in [7] exposes the fact that $p>c_{0} \log \left(1 / c_{1}\right)$, with $c_{0}$ depending on $n$ only.

Lemma 2 and the Gurov-Reshetnyak theorem are continuations of the line of research originating from the John-Nirenberg lemma on BMO spaces.

Lemma 2 is related to the so-called weak reverse Hölder inequalities, which appear in quasiconformal theory and various problems in elliptic PDE's at a more elementary stage than reverse Hölder inequalities and thus their range of applicability is much broader. Various properties and applications of these inequalities, especially to quasiconformal theory, have been discussed in [1], [2], [7], [8]. Important applications of Lemma 2 to quasiconformal theory and PDE's have been described in [7] and [8].

The proof of Lemma 2, presented in [7], relies essentially on a local form of the Fefferman -Stein inequality [3]. In [7] it is presented as follows:

Lemma 3. If $f^{\#} \in L^{p}\left(Q_{0}\right)$ ( $Q_{0}$ a fixed cube), $p \geqq 1$, then $M f \in L^{p}\left(Q_{0}\right)$ and

$$
\left(f_{Q_{0}}|M f|^{p} d x\right)^{1 / p} \leqq 10^{5 n p}\left(f_{Q_{0}}\left|f^{\#}\right|^{p} d x\right)^{1 / p}+10^{n+1} f_{Q_{0}}|f| d x .
$$

Here again the numerical coefficient $10^{5 n p}$ is much too big for large $p$. As a matter of fact the following is true:

Lemma 4. For $f$ as in Lemma $3, p \geqq 1$,

$$
\left(f_{Q_{0}}|M f|^{p} d x\right)^{1 / p} \leqq c_{1} p\left(f_{Q_{0}}\left|f^{\#}\right|^{p} d x\right)^{1 / p}+c_{1} f_{Q_{0}}|f| d x
$$

with $c_{1}$ depending on $n$ only.

Lemma 4 is a direct consequence of an essential refinement of a local Feffer man - Stein inequality obtained by I. Wik [13]. 
Theorem 2 (I. Wik). For an arbitrary cube $Q \subset \boldsymbol{R}^{n}, \quad p>0$, and a function $f \in L^{1}(Q)$ the inequality

$$
\left\|M f-f_{Q}\right\|_{L^{p}(Q)} \leqq c\left\|f^{\#}\right\|_{L^{p}(Q)}
$$

holds with a constant $c \sim c_{0} p b^{1 / p}, c_{0}$ and $b$ independent of $p$ and $Q$.

Wik's method, in particular, gives a Fefferman-Stein inequality with the constant of order $p$ for large $p$, whereas the original Fefferman-Stein method produces a constant of exponential order $2^{p}$. We remark that Wik does not use the Calderón-Zygmund decomposition lemma. Instead, he relies on the direct study of distribution functions and related coverings.

Combining Wik's method with the discussion in [7] we obtain the generalization of the Gurov-Reshetnyak theorem.

Theorem 3. Let $f \in L_{\mathrm{loc}}^{1}(\Omega), 0<\sigma \leqq 1,1 \leqq p<\infty$. Suppose that

$$
f_{\sigma Q}\left|f-f_{\sigma Q}\right| d y \leqq \varepsilon f_{Q}|f| d y
$$

for each cube $Q \subset \Omega$. Then there exists a constant $c$ deperdirg on $n$ only such that $f \in L_{\mathrm{loc}}^{p}(\Omega)$ for every $p<c / \varepsilon$, provided $\varepsilon$ is small. Moreover, for a constant $b$, depending on $\sigma, n$, and $p$ only, we have

$$
\left(f_{Q / 2}|f|^{p} d y\right)^{1 / p} \leqq b f_{Q}|f| d y .
$$

(Instead of factor $1 / 2$ in Theorem 3 any fixed $\sigma_{0}<1$ could be used.) Naturally, a detailed proof gives some explicit estimates for $c$ and $b$, which are omitted here. The order of the optimal integrability exponent $p$, estimated in Theorem 3, is exact.

We note also that Lemma 2 and Theorem 3 have some connections with the results of J. O. Strömberg [12].

All this suggests that many important questions around the Fefferman-Stein inequality, John - Nirenberg BMO lemma and Gurov-Reshetnyak results deserve further study.

Theorem 3 is a refinement of Lemma 2. It implies in particular corresponding improvements of some applications of Lemma 2 discussed in [7] and [8].

The details of the topics discussed above will be presented in an expository paper under preparation and also, hopefully, in the monograph announced in [2]. 


\title{
References
}

[1] Bojarski, B., and T. IwANieC: Some new concepts in the analytical theory of $Q C$ - maps in $\boldsymbol{R}^{n}, n \geqq 3$, and differential geometry. - The Conference on Global Analysis, Garwitz, DDR, October, 1981.

[2] Bojarski, B., and T. IwANIEC: Analytical foundations of the theory of quasiconformal mappings in $\boldsymbol{R}^{n}$. - Ann. Acad. Sci. Fenn. Ser A. I. Math. 8, 1983, 257-324.

[3] Fefferman, C., and E. M. Stein: $H^{p}$ spaces of several variables. - Acta Math. 129, 1972, 137-193.

[4] Gehring, F. W.: The $L^{p}$-integrability of the partial derivatives of a quasiconformal mapping. - Acta Math. 130, 1973, 265-277.

[5] Gurov, L. G.: The stability of Lorentz transformations. Estimates for the derivatives. - Dokl. Akad. Nauk. SSSR 220, 1975, 273-276 (Russian).

[6] Gurov, L. G., and Yu. G. ResetnyaK: A certain analogue of the concept of a function with bounded mean oscillation. - Sibirsk. Mat. Zh. 17, No. 3, 1976, 540-546 (Russian).

[7] IwANIEC, T.: On $L^{p}$-integrability in PDE's and quasiregular mappings for large exponents. Ann. Acad. Sci. Fenn. Ser A. I. Math. 7, 1982, 301-322.

[8] IwANIEC, T.: Some aspects of partial differential equations and quasiregular mappings. - Proceedings of the International Congress of Mathematicians, Warsaw, August 1983, PWN-North-Holland, Waıszawa, 1984.

[9] Muckenhoupt, B.: Weighted norm inequalities for the Hardy maximal function. - Trans. Amer. Math. Soc. 165, 1972, 207-226.

[10] Reshetnyak, Yu. G.: Stability estimates in the class $W_{p}^{1}$ in Liouville's conformal mapping theorem for a closed domain. - Sibirsk. Mat. Zh. 17, 1976, 1382-1394 (Russian).

[11] Reshetnyak, Yu. G.: Stability theorems in geometry and analysis. - Izdat. Nauka, Novosibirsk, 1982 (Russian).

[12] Strömberg, J. O.: Bounded mean oscillation with Orlicz norms and duality of Hardy spaces. Indiana Univ. Math. J. 28, 1979, 511-544.

[13] WIK, I.: A comparison of the integrability of $f$ and $M f$ with that of $f^{\#}$. - Preprint series 2, Department of Mathematics, University of Umeå, 1983.

\author{
Warsaw University \\ Institute of Mathematics \\ 00-901 Warsaw \\ Poland
}

Received 18 September 1984 